\title{
Microvascular endothelial dysfunction is associated with albuminuria and CKD in older adults
}

Stephen L. Seliger ${ }^{1,2^{*}}$, Shabnam Salimi ${ }^{3}$, Valerie Pierre ${ }^{4}$, Jamie Giffuni ${ }^{5}$, Leslie Katzel ${ }^{1,5}$ and Afshin Parsa ${ }^{1,2}$

\begin{abstract}
Background: Impairment in glomerular endothelial function likely plays a major role in the development of albuminuria and CKD progression. Glomerular endothelial dysfunction may reflect systemic microvascular dysfunction, accounting in part for the greater cardiovascular risk in patients with albuminuria. Prior studies of vascular function in CKD have focused on conduit artery function or those with ESRD, and have not examined microvascular endothelial function with albuminuria.
\end{abstract}

Methods: We conducted a cross-sectional study among older hypertensive male veterans with stage 1-4 CKD, and hypertensive controls without CKD. Microvascular function was quantified by two distinct Laser-Doppler flowmetry (LDF) measures: peak responses to 1) post-occlusive reactive hyperemia (PORH) and 2) thermal hyperemia (TH), measured on forearm skin. Associations of each LDF measure with albuminuria, eGFR, and CKD status were estimated using correlation coefficients and multiple linear regression, accounting for potential confounders.

Results: Among 66 participants (mean age 69.2 years), 36 had CKD (mean eGFR $46.1 \mathrm{cc} / \mathrm{min} / 1.73 \mathrm{~m}^{2} ; 30.6 \%$ with overt albuminuria). LDF responses to PORH and TH were 43 and $39 \%$ significantly lower in multivariate analyses among those with macroalbuminuria compared to normoalbuminuria, $(\beta=-0.42, p=0.009$ and $\beta=-0.37, p=0.01$, respectively). Those with CKD had a $23.9 \%$ lower response to PORH compared to controls ( $p=0.02$ after adjustment). In contrast, $\mathrm{TH}$ responses did not differ between those with and without CKD.

Conclusions: Microvascular endothelial function was strongly associated with greater albuminuria and CKD, independent of diabetes and blood pressure. These findings may explain in part the excess systemic cardiovascular risk associated with albuminuria and CKD.

Keywords: Endothelial function, CKD, Albuminuria, Vascular disease, Blood pressure, Laser Doppler flowmetry

\section{Background}

The risk of cardiovascular related morbidity and mortality in individuals with chronic kidney disease (CKD) is exceedingly high and often greater than the risk of progressing to end-stage renal disease (ESRD), especially in older adults [1]. However, the mechanisms which account for the systematic vascular manifestations of albuminuria and CKD are not fully elucidated. The endothelium, a single cell layer providing the interface between circulating blood and the vascular wall, regulates many critical aspects of vascular

\footnotetext{
* Correspondence: sseliger@medicine.umaryland.edu

'Department of Medicine, University of Maryland School of Medicine, $22 \mathrm{~S}$.

Greene Street, Room N3W143, Baltimore, MD 21201, USA

${ }^{2}$ Medicine, VA Maryland Healthcare System, Baltimore, USA

Full list of author information is available at the end of the article
}

function including vascular tone, coagulation, and protection against oxidative stress and inflammation [2]. It has been proposed that elevated urine albumin excretionespecially low to moderate elevations - represents a state of generalized endothelial dysfunction, but there has been relatively limited direct evidence in humans to support this hypothesis [3]. Moreover, dysfunction of the endothelium is increasingly recognized as a sentinel event in the development and progression of both focal and systemic vascular disease. Indeed, markers and measures of endothelial dysfunction, a determinant of systemic vasculopathy, have been shown to be increased in CKD [4]. However, a variety of endothelial function measures have been employed, each with advantages and weaknesses. Most prior studies 
examining endothelial function have focused on ischemic flow mediated dilatation (FMD) of medium sized conduit arteries such as the brachial artery. These studies have demonstrated a significant impairment in the endotheliumdependent vasodilatation of the brachial artery among patients with ESRD, late stage CKD, or patients with glomerulonephritis, compared to controls [5-9]. Although such conduit artery function represents an important potential mediator of vascular risk, the renal vascular bed consists predominately of micro-vessels. Therefore, dysfunction of the microvascular endothelium may better characterize the systemic vasculopathy of CKD $[10,11]$. Studies of endothelial function and albuminuria have been less consistent, in the context of variable methodological approaches and populations and have not focused on albuminuria in patients with HTN with and without moderate CKD [8, 1215]. Non-invasive evaluation of the cutaneous microcirculatory is readily feasible using the technique of Laser- Doppler flowmetry (LDF). Specifically, the semi-automated use of LDF to measure cutaneous microcirculatory function in response to specific provocations has been advocated as a practical and reliable in-vivo assay of systemic microvascular vasodilatory function [16, 17]. A small number of prior studies using LDF techniques have demonstrated deficits in endothelium-dependent microvascular function in patients with ESRD and advanced non-dialysis dependent CKD compared to controls, and an association of these functional measures with cardiovascular risk [10, 18-20]. However, it is unclear whether microvascular endothelial dysfunction is present among those with earlier stages of CKD, and whether common comorbidities in those with CKD such as hypertension and diabetes account for this dysfunction.

We therefore performed an observational, crosssectional study to examine the relationship of microvascular endothelial function with non-dialysis CKD and measures of renal disease severity (albuminuria and eGFR) among older adults with treated hypertension. We hypothesized that those older adults with greater albuminuria and lower eGFR would have more diminished endothelial function as assessed by two separate laser Doppler flowmetry based measures, after accounting for demographic and co-morbid factors.

\section{Methods}

\section{Study subjects}

Community-dwelling hypertensive outpatients at the VA Maryland Healthcare System aged 60 or older with and without chronic kidney disease (stages 1-4) or diabetes mellitus were eligible for participation. CKD was defined according to K/DOQI criteria as persistent eGFR $<60 \mathrm{cc} /$ $\min / 1.73 \mathrm{~m}^{2}$ or abnormal albuminuria for at least 3 months. Patients with any of the following conditions were excluded from participation: NYHA class III-IV heart failure; coronary revascularization or acute coronary syndrome within the prior 3 months; vascular claudication; prior stroke; dementia; severe anemia $(\mathrm{Hb}<9 \mathrm{~g} / \mathrm{dL})$, and poorly controlled hypertension $(\mathrm{BP}>180 / 95)$ or diabetes (HbA1C $>11 \%)$. Participants were recruited from nephrology, diabetes, and primary care clinics. Participants were enrolled consecutively from $11 / 2010$ through $2 / 2013$, without specific matching of CKD and non-CKD patients.

\section{Covariates and measurements}

Clinical co-morbidity was determined through review of the electronic medical record and participant interview. Coronary artery disease was defined as a history of prior myocardial infarction, coronary revascularization, and/or coronary disease by angiography. Albuminuria was quantified with the urine albumin/creatinine ratio on a spot morning urine sample after an overnight fast. Albuminuria was classified as per standard criteria as normoalbuminuria if albumin/creatinine ratio $<30 \mathrm{mg} / \mathrm{gm}$; microabluminuria if albumin/creatinine ratio was 30 to $300 \mathrm{mg} / \mathrm{gm}$ and macroalbuminuria if albumin/creatinine ratio was $>300 \mathrm{mg} / \mathrm{gm}$. Blood pressure was recorded in the sitting position prior to LDF measurements using a Critikon Dinamap 1846 SX monitor (GE Medical Systems), and mean arterial pressure calculated as (DBP + (SBP-DBP)/3). GFR was estimated from serum creatinine measured with an IDMS-traceable assay using the 4-variable MDRD equation.

\section{Laser Doppler flowmetry (LDF)}

LDF uses a laser light source and Doppler shift effect to non-invasively measure microvascular blood flow on a relative BPU (Blood Perfusion Units) scale. This allows for the measurement of relative change in microcirculatory perfusion in response to various interventions. All measurements were performed according to a standardized protocol with a Perimed, PF5020 unit in a temperature-controlled room after an overnight fast and after withholding of AM doses of vasoactive medications including nitrates. Active smokers were instructed to abstain from smoking the day of LDF testing. We used two distinct LDF based measures: 1) post occlusive reactive hyperemic (PORH) and 2) thermal hyperemic (TH) mediated response. In TH-LDF, the LDF probe is placed on the volar aspect of the forearm and heated to $44 \mathrm{C}$ causing dilatation of the skin vessels and increased flow, which is measured over $30 \mathrm{~min}$. TH-LDF results in a biphasic response, an initial axon reflex peak phase which is mediated in part by the neuropeptides substance $\mathrm{P}$ and calcitonin gene-related peptide (CGRP). This is followed by a more prolonged plateau phase, largely mediated by the endothelial release of nitric oxide [21]. For PORH-LDF, the upper arm flow is occluded by a blood 
pressure cuff for a 4-min period. The resultant ischemia induces dilatation of resistance vessels. Upon release of the cuff, there is a rush of blood flow secondary to the induced decreased arterial resistance, which in turn causes increase shear stress on the vessels, inducing further dilatation by endothelial and potentially nonendothelial based mediators. All blood flow tracings were digitally transferred and interpreted by a single reviewer (AS) blinded to clinical characteristics of the participant. Tracings with technical deficiencies precluding accurate quantification (eg participant motion, displacement of probe during recording) were excluded from analysis. The percent increase in flow from baseline is then used as a measure of endothelial function and vascular reactivity for both $\mathrm{TH}$ and PORH-LDF measures [21]. Additionally, PORH- and TH-LDF were categorized as high or low, if above or below the median for each measure, respectively.

\section{Statistical methods}

Clinical characteristics between those with and without CKD were compared using the independent-sample $t$-tests for continuous variables and chi-squared or Fisher's exact test for categorical variables. Comparisons of each LDF measure across albuminuria category were performed with ANOVA tests. Correlations of each LDF measure (response to $\mathrm{TH}$ and $\mathrm{PORH}$ ) with albuminuria were estimated using Pearson correlation coefficients, after natural logarithm (lntransformation) of the urine albumin/creatinine ratio to ensure a normal distribution. The association of albuminuria and CKD with each LDF measure (dependent variable) after adjusting for demographic and co-morbid factors was estimated using multiple linear regression. Adjustment covariates were selected a priori and included age, self-reported race (African-American vs. other), mean arterial pressure, coronary artery disease, and diabetes. Associations were presented as standardized regression coefficients $(\hat{\beta})$ to allow comparison across different outcome measurements. The Cook-Weisberg test was used to confirm the assumption of homoscedasticity.

\section{Results}

Cutaneous PORH and/or TH provocations measures were obtained on 66 male participants. Of these, 61 PORH and $52 \mathrm{TH}$ tracing were determined to be of sufficient technical quality for quantification of peak response. Compared to those without CKD $(N=30)$, those with CKD $(N=36)$ were overall well matched with similar age, race, blood pressure, body mass index, use of renin-angiotensin antagonists, and smoking history, but were more likely to have diabetes $(p<$ 0.01) (Table 1.) Of those with CKD, 11 (30.6\%) had overt albuminuria, with mean (SD) eGFR ${ }_{M D R D} 46.1(2.4) \mathrm{cc} / \mathrm{min} /$
Table 1 Baseline characteristics of study sample

\begin{tabular}{llll}
\hline & No CKD $(N=30)$ & CKD (N=36) & $p$-value \\
\hline Age & $68.6(1.4)$ & $69.7(1.2)$ & 0.5 \\
African American & $18(60 \%)$ & $18(50 \%)$ & 0.7 \\
SBP $(\mathrm{mmHg})$ & $137.6(19.2)$ & $143.1(16.6)$ & 0.2 \\
DBP $(\mathrm{mmHg})$ & $74.0(10.5)$ & $74.8(8.7)$ & 0.7 \\
BMI & $30.4(5.5)$ & $31.0(6.3)$ & 0.7 \\
Diabetes & $6(20 \%)$ & $27(75.0 \%)$ & $<0.01$ \\
Coronary Artery Disease ${ }^{a}$ & $2(6.7 \%)$ & $8(22.2 \%)$ & 0.1 \\
Smoking & & & \\
$\quad$ Current & $0(0 \%)$ & $1(2.8 \%)$ & 0.5 \\
$\quad$ Former & $21(70 \%)$ & $21(58.3 \%)$ & \\
$\quad$ Never & $9(30 \%)$ & $13(36 \%)$ & \\
ACEl or ARB & $20(67 \%)$ & $28(77.8 \%)$ & 0.3 \\
Nitrates & 0 & 2 & 0.3 \\
eGFR (cc/min/1.73 m2) & $85.4(19.2)$ & $46.1(2.4)$ & \\
Alb/Cr ratio & $3.8[2.9,7.8]$ & $83.3[16.6,367.0]$ & \\
\hline
\end{tabular}

Cell values represent mean (SD), median [inter-quartile range], and $\mathrm{N}(\%)$

a Patients with unstable coronary disease or with recent coronary intervention were excluded

$1.73 \mathrm{~m}^{2}$; 4 (11 \%) had stage 2, 28 (78 \%) had stage 3, and 4 (11\%) had stage 4 CKD.

\section{PORH-LDF}

Comparing those with high vs. low PORH-LDF, those with lower post-occlusive microvascular reactivity had modestly lower systolic blood pressure and significantly lower eGFR and higher albuminuria (Table 2.) In univariate analysis, PORH-LDF was $43 \%$ lower among those with macroalbuminuria compared to those with normoalbuminuria (Fig. 1a; $95 \% \mathrm{CI}=0.10$ to $0.90 \%, p=0.03$ ) and was inversely correlated with albuminuria $(\mathrm{r}=-0.31, p=0.01)$ (Fig. 2a). After adjustment for demographic factors, blood pressure and diabetes, PORH-LDF remained inversely associated with greater albuminuria (Table $3 ; \hat{\beta}=-0.37,95 \% \mathrm{CI}=-0.55$ to $-0.08, p=0.01$.) The association between PORH and albuminuria did not differ with diabetic status (all tests of interaction, $p>0.5$ ). eGFR as a continuous variable was not associated with PORH-LDF measures $(p=0.2$.) Among those with CKD, PORH-LDF response was $24 \%$ lower compared to non-CKD controls (Fig. 3a: $95 \% \mathrm{CI}=-0.61 \%$ to $-0.05 \%, p=0.045)$. After multivariate adjustment, this association remained significant (Table $3 ; \hat{\beta}=-0.38,95 \% \mathrm{CI}$ $=-0.62$ to $-0.01, p=0.02$ ).

\section{TH-LDF}

Consistent with our PORH-LDF based results, those with lower $\mathrm{TH}$ reactivity had higher albuminuria (Table 2; $p=$ $0.009)$. Albuminuria was inversely and strongly correlated with TH-LDF (Fig. 2b:r $=-0.41, p=0.003$.) TH-LDF 
Table 2 Characteristics of study sample, by Post-Occlusive and Thermal Hyperemic categories

\begin{tabular}{|c|c|c|c|c|c|c|}
\hline & \multicolumn{3}{|c|}{ Post-Occlusive Reactive Hyperemia $(N=65)$} & \multicolumn{3}{|c|}{ Thermal Hyperemia $(N=52)$} \\
\hline & High & Low & $p$-value & High (>892 \%) & Low (<892\%) & $p$-value \\
\hline Age & 68.6 & $70.6(7.6)$ & 0.3 & $68.5(7.3)$ & $69.8(1.5)$ & 0.5 \\
\hline African American & $15(48.4 \%)$ & $17(56.7 \%)$ & 0.5 & $17(65.4 \%)$ & $12(46.2 \%)$ & 0.2 \\
\hline $\mathrm{SBP}(\mathrm{mmHg})$ & $145.5(15.0)$ & $136.0(18.3)$ & 0.03 & $141.3(16.0)$ & $137.2(18.8)$ & 0.4 \\
\hline $\mathrm{DBP}(\mathrm{mmHg})$ & $76.5(10.0)$ & $72.1(8.1)$ & 0.06 & $75.4(10.2)$ & $73.2(8.1)$ & 0.4 \\
\hline $\mathrm{BMl}$ & $30.7(6.6)$ & $30.4(5.5)$ & 0.9 & $30.2(6.3)$ & $32.6(4.8)$ & 0.14 \\
\hline Diabetes & $13(41.9 \%)$ & $18(60 \%)$ & 0.2 & $16(61.5 \%)$ & $10(38.5 \%)$ & 0.10 \\
\hline Coronary Artery Disease ${ }^{a}$ & $5(16.1 \%)$ & $5(16.7 \%)$ & 1.0 & $4(15.4 \%)$ & $4(15.4 \%)$ & 1.0 \\
\hline Smoking & & & & & & 0.6 \\
\hline Current & $19(61.3 \%)$ & $19(63.3 \%)$ & 0.6 & 1 (3.9\%) & $0(0 \%)$ & \\
\hline Former & $0(0 \%)$ & 1 (3.3 \%) & & $15(57.7 \%)$ & $17(65.4 \%)$ & \\
\hline Never & $12(38.7 \%)$ & $9(30 \%)$ & & $10(38.5 \%)$ & 9 (34.6 \%) & \\
\hline ACEI or ARB & $19(63.3 \%)$ & $26(83.9 \%)$ & 0.07 & $18(69 \%)$ & 18 (69 \%) & 1.0 \\
\hline Nitrates & 1 (3.3\%) & 1 (3.3 \%) & 0.8 & 1 (3.9\%) & 0 & 0.5 \\
\hline eGFR (cc/min/1.73 m2) & $69.2(4.9)$ & $56.0(4.1)$ & 0.04 & $61.9(24.0)$ & $68.7(29.8)$ & 0.4 \\
\hline \multirow[t]{2}{*}{ Alb/Cr ratio } & $5[2.3,26]$ & 18.2 & 0.006 & 4.4 & 21.5 & 0.009 \\
\hline & & {$[7.2,334]$} & & {$[2.8,11.8]$} & {$[4.4,207]$} & \\
\hline
\end{tabular}

Cell values represent mean (SD), median [inter-quartile range], and $\mathrm{N}(\%)$

${ }^{\text {a }}$ Patients with unstable coronary disease or with recent coronary intervention were excluded

measures were $39 \%$ lower among those with macroalbuminuria compared to normoalbuminuria (Fig. 1b; $p=$ 0.03.) After adjustment for demographic factors, and major co-morbidities, greater albuminuria was associated with lower TH-LDF measures (Table 3.) Of note, the association of TH-LDF measures with albuminuria $(\hat{\beta}=-0.42)$ was markedly greater than the associations of age $(\beta=0.15)$ and mean arterial pressure $(\beta=0.08)$.

In contrast with PORH-LDF, there was no significant association of CKD or eGFR with TH-LDF based measures (Fig. $3 \mathrm{~b}$ and Table $3 ; p=0.6$ and $p=0.9$, respectively.)

The relationship between LDF responses with albuminuria did not differ between those with and without diabetes in either PORH- or TH-LDF $(p>0.5)$.

\section{Discussion}

Among older hypertensive men with and without non-dialysis dependent CKD, microvascular endothelial function as estimated by the cutaneous hyperemic response to brachial artery occlusion and localized heating is decreased in a graded, dose-dependent fashion with greater albuminuria. Furthermore, those with CKD had a $24 \%$ lower PORH response than those without CKD. These associations were robust to adjustment for selected potential confounders including blood pressure, and were qualitatively greater than the associations of other factors such as greater age and blood pressure.
Even low-level elevations of albuminuria are associated with measureable increases in risk of vascular disease, including myocardial infarction, stroke, cardiovascular death, and cognitive decline, especially in older adults. While podocyte function has long been recognized as a key component of the glomerular albumin barrier, there has been continued evidence demonstrating that not all proteinuria can be accounted for based on podocyte dysfunction, such as in early diabetic nephropathy and systemic inflammatory states (eg, sepsis and inflammatory bowel disease). Recent evidence strongly suggests a prominent role for the glomerular endothelial layer and associated glycocalyx as a key barrier to albumin across the glomerular basement membrane [22]. This finding would potentially account in part for the strong association between albuminuria and cardiovascular disease risk, independent of decreased GFR. The results of this study provide support for this hypothesis, suggesting that at least in older adults with hypertension - microvascular endothelial function is a major correlate of albuminuria, independent of blood pressure, diabetes and decreased GFR.

Prior studies using LDF to measure microvascular function in CKD patients have primarily focused on those with ESRD [10,11, 18, 20, 23], whether dialysisdependent or recipients of renal transplants. These studies have generally reported significantly lower endothelial function in ESRD or advanced CKD patients [19] compared to controls, although there have been discrepant results regarding whether these differences are independent of hypertension [18]. Prior studies in 

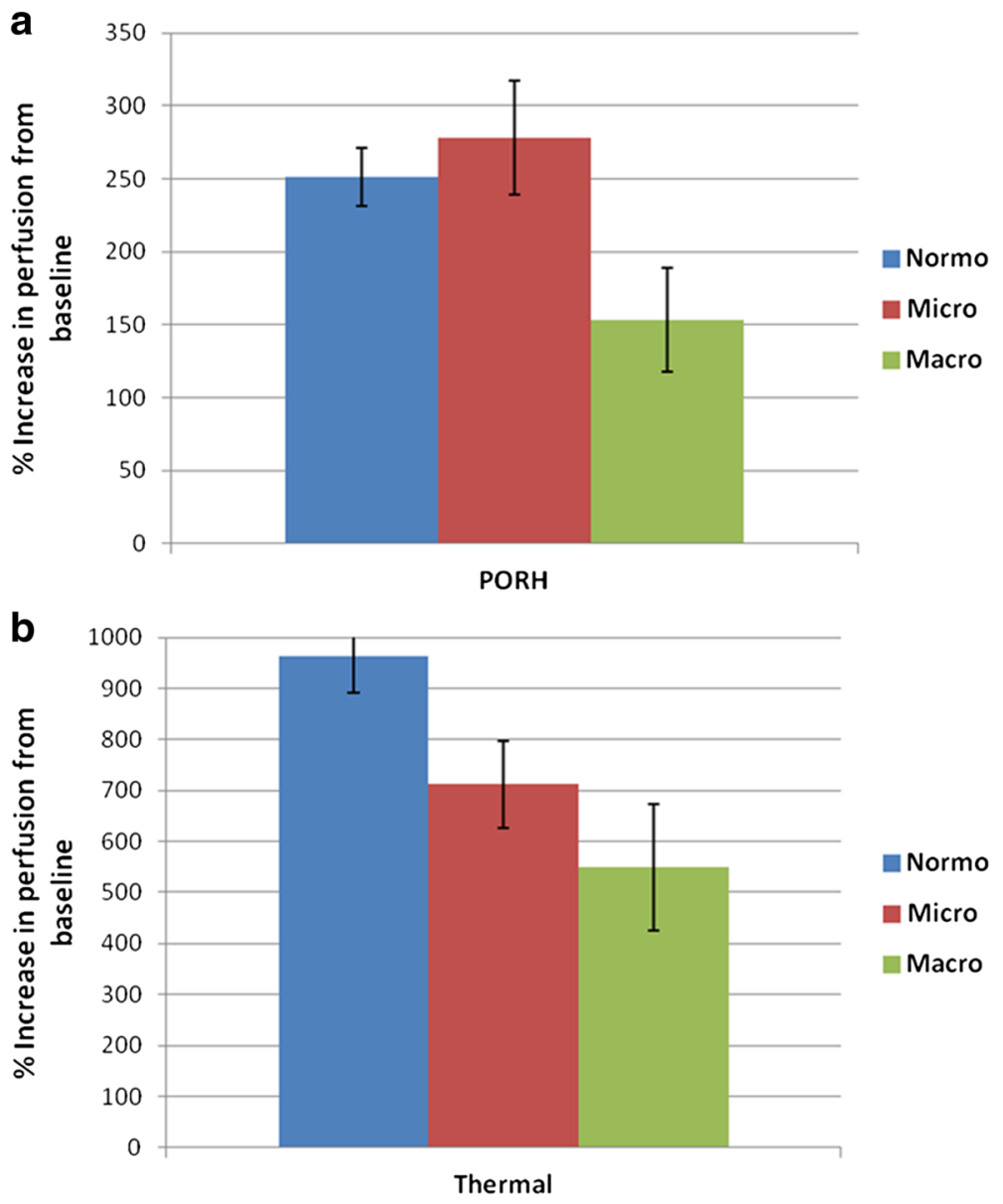

Fig. 1 Hyperemic response to post-occlusive (a) and thermal (b) provocations, by albuminuria category

ESRD have also suggested that these measures are prognostically important for short-term prediction of cardiovascular events in dialysis-dependent patients [11] and for allograft failure in renal transplant recipients [23]. Moreover, since various measures of endothelial function and vascular reactivity are only modestly correlated to each other as they capture various components of endothelial and vascular response [21], we used two methods, TH and PORH to better ascertain microvascular responses. This allowed us to estimate the association between CKD and PORH, which may have been missed by using only $\mathrm{TH}$ based measures, extending previous findings of reduced microvascular endothelial function to those with mild-moderate CKD. Lastly, by comparing CKD patients to age matched controls with hypertension, as opposed to healthy controls without any comorbidity, our findings suggest that the endothelial functional impairment is not explained merely by the increased prevalence of hypertension in CKD or diabetes.
Previous studies of brachial FMD and/or LDF based measures of vascular and endothelial function have yielded conflicting results regarding associations with proteinuria. Most notably, many of these were in patients with diabetes and did not explore association between endothelial function measures and proteinuria, independent of diabetes status. Several involved patients with non-diabetic and nonhypertensive glomerulonephritis or patients with HIV, which cannot be generalized to the patient with hypertension-attributed CKD. Our findings of strong consistent dose dependent association between two distinct measures of microvascular endothelial function and reactivity, independent of blood pressure and diabetes, meaningfully extend previous findings. Moreover, while obtaining reliable brachial artery FMD measures can be technically challenging and costly [24], the semi-automated LDF based $\mathrm{TH}$ and PORH measures of skin microvascular reactivity require minimal training, are relatively easy to obtain and have good reproducibility and may at times be preferred over other non-invasive measures of endothelial function and vascular reactivity [25-27]. Furthermore, LDF 

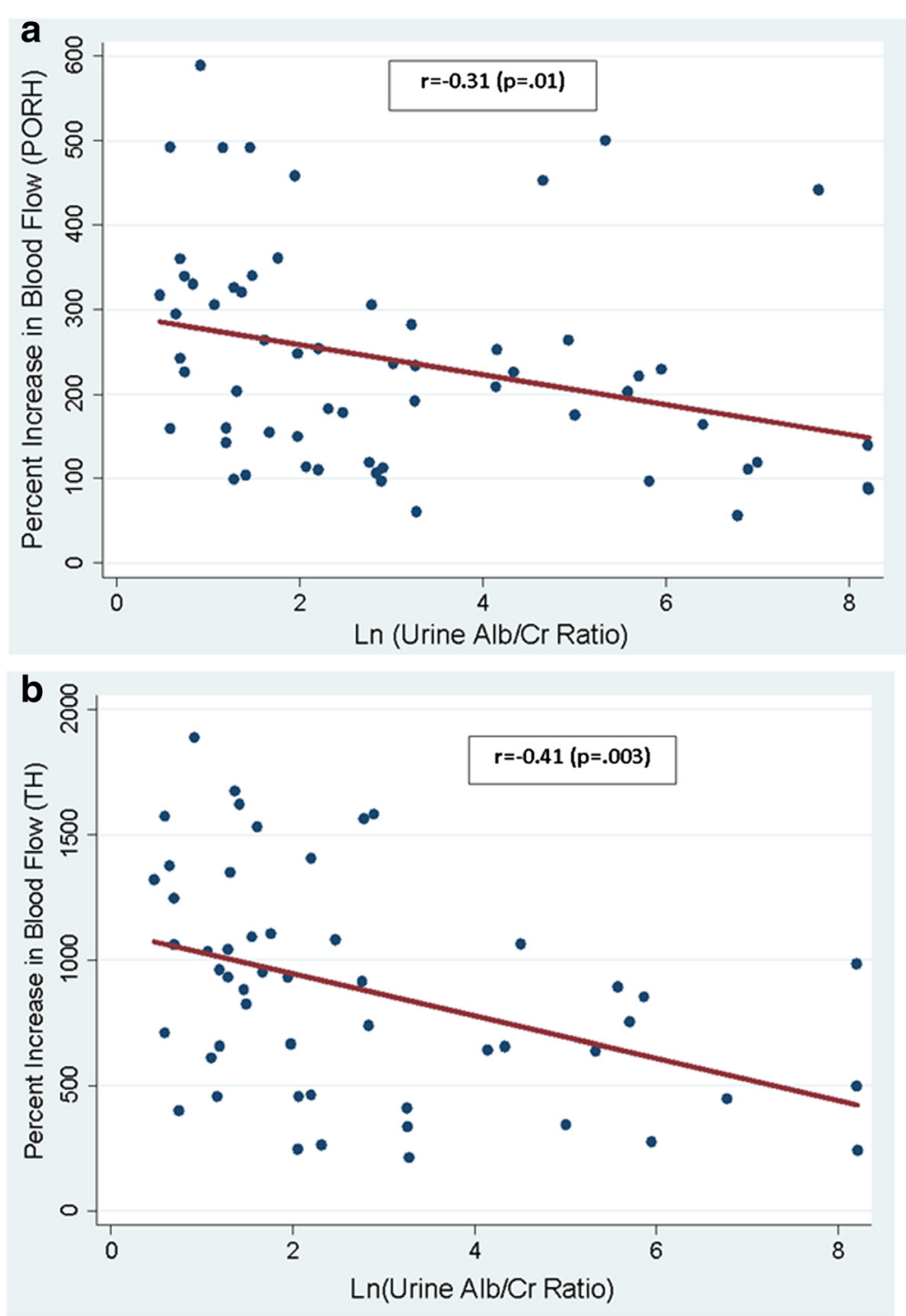

Fig. 2 Association of hyperemic response to post-occlusive (a) and (b) thermal provocations with albuminuria

measures are considered to be less dependent on systemic arterial blood pressure, as they reflect blood flow difference in the microcirculatory vessels, which are not subject to the high pressure in conduit vessels such as the brachial artery, and further represent difference in flow from the baseline, which would normalize any BP-related difference in baseline blood flow.

Limitations of the present study include the modest sample size, which precluded extensive subgroup analysis and limited the potential number of confounders

Table 3 Association $(\hat{\beta})$ of microvascular endothelial function with CKD, albuminuria, and eGFR

\begin{tabular}{|c|c|c|c|c|}
\hline & \multicolumn{2}{|c|}{ Post-Occlusive Hyperemia $(N=61)$} & \multicolumn{2}{|c|}{ Thermal Hyperemia $(N=52)$} \\
\hline & Unadjusted & Adjusted & Unadjusted & Adjusted \\
\hline Albuminuria & $-0.31(p=0.01)$ & $-0.37(p=0.01)$ & $-0.41(p=0.003)$ & $-0.42(p=0.009)$ \\
\hline CKD (vs. no CKD control) & $-0.25(p=-0.045)$ & $-0.38(p=0.02)$ & $-0.08(p=0.6)$ & $0.09(p=0.6)$ \\
\hline eGFR & $0.17(p=0.2)$ & $0.20(p=0.2)$ & $0.12(p=0.41)$ & $-0.02(p=0.9)$ \\
\hline
\end{tabular}

Albuminuria: Natural log- transformed Urine albumin to creatinine ratio, Cell values represent standardized regression coefficient Adjusted for age, race (African-American vs. other), mean arterial blood pressure, diabetes, and coronary artery disease 

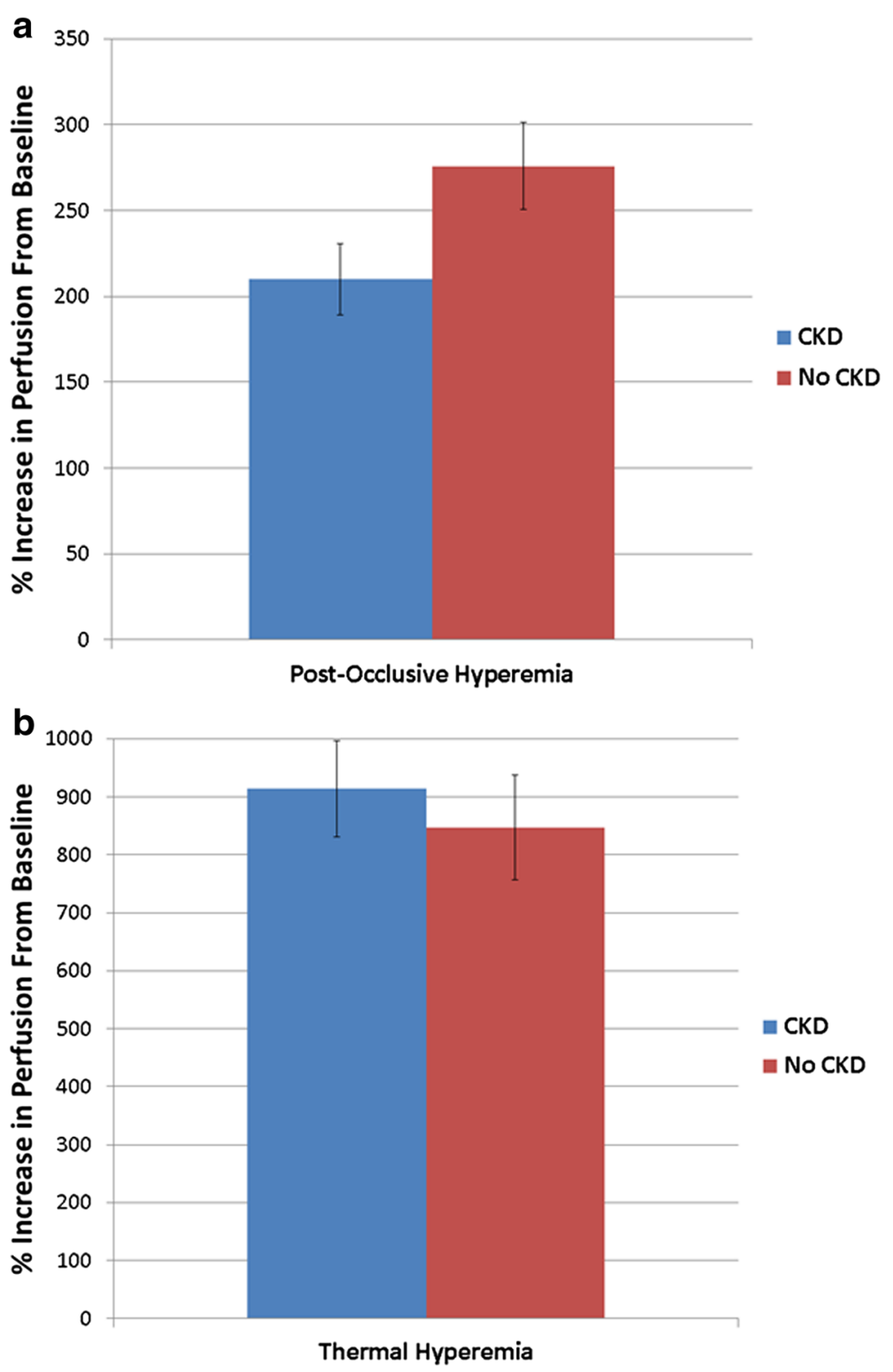

Fig. 3 Hyperemic response to post-occlusive (a) and thermal (b) provocations, by albuminuria category, among those with and without CKD

that could be considered in multivariate models. Although those without CKD were of similar age, ethnic background, and blood pressure to those with CKD, they were considerably less likely to have diabetes and stable coronary heart disease. Although we adjusted for these differences in regression models, residual confounding may have persisted. The study population consisted of older men-consistent with the recruitment setting of a VA medical center - and the results may not apply to women or middle-aged adults. Due to the cross-sectional design of the study, we cannot determine whether microvascular dysfunction is a causal factor for albuminuria and CKD, or a result of the renal disease.

\section{Conclusions}

In conclusion, we find that LDF-based measures of endothelial function and microvascular reactivity are the strongest determinants of albuminuria in our older hypertensive population of patients with and without CKD, irrespective of diabetes status. We also found our patients with CKD to have lower microvascular reactivity based on our PORH measures. These findings highlight that skin measures of microvasculatory endothelial function and reactivity as a proxy of systemic endothelial function are strongly correlated with albuminuria and that albuminuria may plausibly reflect glomerular microvasculatory dysfunction. This provides further evidence in favor of the 
hypothesis that endothelial function comprises a common pathophysiology linking renal dysfunction and albuminuria with systemic vascular disease.

\section{Abbreviations}

CKD, chronic kidney disease; ESRD, end-stage renal disease; FMD, flow-mediated dilatation; LDF, laser Doppler flowmetry; PORH, post-occlusive reactive hyperemia; $\mathrm{TH}$, thermal hyperemia

\section{Acknowledgements}

None.

\section{Funding}

Research supported by funding from VA Rehabilitation Research \& Development (SS, LK), National Institutes of Health/NIDDK T35DK095737 (VP), the National Institute on Aging Claude D. Pepper Older Americans Independence Center (P30-AG028747), T32 AG00262, and the Baltimore VAMC Geriatric Research Education and Clinical Center (GRECC).

\section{Availability of data and materials}

All data utilized for these reports are stored by Dr. Seliger at the Baltimore VA Medical Center on a secured research sever, in compliance with Veterans Health Administration information security policies. As this data is part of ongoing analyses, and is subject to the information security policies referenced above, it is not the intent of the authors to share these data directly at this time. The authors welcome however invitations for collaborations utilizing these data.

\section{Authors' contributions}

SLS conceived of the study, participated in design and acquisition of the data and data analysis, and drafted the manuscript. SS participated in data analysis and interpretation, and critically revised the manuscript. VP participated in data analysis and interpretation and critically revised the manuscript. JG participated in data collection and critically revised the manuscript. LK helped conceived of the study, participated in study design, participated in data interpretation, and critically revised the manuscript. AP participated in data collection and interpretation, and assisted with drafting and critical revision of the manuscript. All authors read and approved the final manuscript.

\section{Competing interests}

The authors declare that they have no competing interests.

\section{Consent for publication}

All authors have provided consent to publish.

\section{Ethics approval and consent to participate}

All participants gave written informed consent for participation. The protocol was reviewed and approved by the University of Maryland, Baltimore IRB and the VA Maryland Healthcare System Research and Development Committee.

\section{Author details}

${ }^{1}$ Department of Medicine, University of Maryland School of Medicine, 22 S. Greene Street, Room N3W143, Baltimore, MD 21201, USA. ${ }^{2}$ Medicine, VA Maryland Healthcare System, Baltimore, USA. ${ }^{3}$ Epidemiology and Public Health, University of Maryland School of Medicine, Baltimore, USA.

${ }^{4}$ Creighton University School of Medicine, Omaha, USA. ${ }^{5}$ GRECC, VA Maryland Healthcare System, Baltimore, USA.

Received: 18 June 2015 Accepted: 7 July 2016

\section{Published online: 13 July 2016}

\section{References}

1. Keith DS, Nichols GA, Gullion CM, Brown JB, Smith DH. Longitudinal followup and outcomes among a population with chronic kidney disease in a large managed care organization. Arch Intern Med. 2004;164:659-63.

2. Deanfield JE, Halcox JP, Rabelink TJ. Endothelial function and dysfunction: testing and clinical relevance. Circulation. 2007;115:1285-95.

3. Satchell S. The role of the glomerular endothelium in albumin handling. Nat Rev Nephrol. 2013;9:717-25.
4. Malyszko J. Mechanism of endothelial dysfunction in chronic kidney disease. Clin Chim Acta. 2010:411:1412-20.

5. Yildiz A, Oflaz H, Pusuroglu $H$, et al. Left ventricular hypertrophy and endothelial dysfunction in chronic hemodialysis patients. Am J Kidney Dis. 2003:41:616.

6. Yilmaz MI, Saglam M, Caglar K, et al. The determinants of endothelial dysfunction in CKD: oxidative stress and asymmetric dimethylarginine. Am J Kidney Dis. 2006;47:42-50.

7. Ghiadoni L, Cupisti A, Huang Y, et al. Endothelial dysfunction and oxidative stress in chronic renal failure. J Nephrol. 2004:17:512-9.

8. Paisley KE, Beaman M, Tooke JE, Mohamed-Ali V, Lowe GD, Shore AC. Endothelial dysfunction and inflammation in asymptomatic proteinuria. Kidney Int. 2003;63:624-33.

9. Caglar K, Yilmaz Ml, Saglam M, et al. Short-term treatment with sevelamer increases serum fetuin-a concentration and improves endothelial dysfunction in chronic kidney disease stage 4 patients. Clin J Am Soc Nephrol. 2008:3:61-8.

10. Stewart J, Kohen A, Brouder D, et al. Noninvasive interrogation of microvasculature for signs of endothelial dysfunction in patients with chronic renal failure. Am J Physiol Heart Circ Physiol. 2004;287:H2687-96.

11. Kruger A, Stewart J, Sahityani R, et al. Laser Doppler flowmetry detection of endothelial dysfunction in end-stage renal disease patients: correlation with cardiovascular risk. Kidney Int. 2006;70:157-64

12. Gupta SK, Shen C, Mather KJ, Agarwal R, Dube MP. Neither proteinuria nor albuminuria is associated with endothelial dysfunction in HIV-infected patients without diabetes or hypertension. J Infect Dis. 2011;204:1946-50.

13. Taslipinar A, Yaman H, Yilmaz MI, et al. The relationship between inflammation, endothelial dysfunction and proteinuria in patients with diabetic nephropathy. Scand J Clin Lab Invest. 2011;71:606-12.

14. Yilmaz MI, Sonmez A, Saglam M, et al. ADMA levels correlate with proteinuria, secondary amyloidosis, and endothelial dysfunction. J Am Soc Nephrol. 2008;19:388-95.

15. Yilmaz Ml, Saglam M, Qureshi AR, et al. Endothelial dysfunction in type-2 diabetics with early diabetic nephropathy is associated with low circulating adiponectin. Nephrol Dial Transplant. 2008:23:1621-7.

16. Holowatz LA, Thompson-Torgerson CS, Kenney WL. The human cutaneous circulation as a model of generalized microvascular function. J Appl Physiol. 2008;105:370-2

17. Yvonne-Tee GB, Rasool AH, Halim AS, Rahman AR. Reproducibility of different laser Doppler fluximetry parameters of postocclusive reactive hyperemia in human forearm skin. J Pharmacol Toxicol Methods. 2005:52:286-92.

18. Cupisti A, Rossi M, Placidi S, et al. Responses of the skin microcirculation to acetylcholine in patients with essential hypertension and in normotensive patients with chronic renal failure. Nephron. 2000:85:114-9.

19. Thang $\mathrm{OH}$, Serne $\mathrm{EH}$, Grooteman MP, et al. Premature aging of the microcirculation in patients with advanced chronic kidney disease. Nephron Extra. 2012;2:283-92.

20. Wilkinson SP, Spence VA, Stewart WK. Arterial stiffening and reduced cutaneous hyperaemic response in patients with end-stage renal failure. Nephron. 1989:52:149-53.

21. Cracowski JL, Minson CT, Salvat-Melis M, Halliwill JR. Methodological issues in the assessment of skin microvascular endothelial function in humans. Trends Pharmacol Sci. 2006;27:503-8.

22. Abularrage CJ, Sidawy AN, Aidinian G, Singh N, Weiswasser JM, Arora S. Evaluation of the microcirculation in vascular disease. J Vasc Surg. 2005:42:574-81.

23. Dahle DO, Midtvedt K, Hartmann A, et al. Endothelial dysfunction is associated with graft loss in renal transplant recipients. Transplantation. 2013:95:733-9.

24. Bots ML, Westerink J, Rabelink TJ, de Koning EJ. Assessment of flow-mediated vasodilatation (FMD) of the brachial artery: effects of technical aspects of the FMD measurement on the FMD response. Eur Heart J. 2005;26:363-8.

25. Roustit M, Cracowski JL. Non-invasive assessment of skin microvascular function in humans: an insight into methods. Microcirculation. 2012;19:47-64.

26. Holowatz LA, Thompson-Torgerson CS, Kenney WL. The human cutaneous circulation as a model of generalized microvascular function. J Appl Physiol (1985). 2008;105:370-2

27. Shamim-Uzzaman QA, Pfenninger D, Kehrer C, et al. Altered cutaneous microvascular responses to reactive hyperaemia in coronary artery disease: a comparative study with conduit vessel responses. Clin Sci (Lond). 2002;103: 267-73. 